\title{
The Effect of Mucolytic Agents and Topical Decongestants on the Ciliary Activity of Chicken Tracheal Organ Cultures
}

\author{
JAMES P. DUDLEY AND JAMES D. CHERRY ${ }^{(1)}$ \\ Division of Infectious Dise'ases, Department of Pediatrics, Center for Health Sciences, University of California at \\ Los Angeles, Los Angeles, California, USA
}

\begin{abstract}
Summary
The chicken tracheal organ culture system was used to study the effect on cilia function of two frequently used topical decongestants and four mucolytic agents. The agents used were: phenylephrine hydrochloride spray $0.25 \%$, oxymetazoline $0.05 \%$, sodium bicarbonate $7.5 \%$, acetyleysteine $10 \%$, pancreatic dornase $\mathbf{5 0 , 0 0 0}$ units $/ \mathrm{ml}$, and $\mathrm{L}$-arginine $5.6 \%$. The tracheal rings were given two kinds of exposure to the test drugs. Only pancreatic dornase was not ciliotoxic. Until further data are available, including studies with human organ cultures, it would perhaps be wise to discourage the topical use of phenylephrine hydrochloride spray $0.25 \%$ and oxymetazoline $0.05 \%$, and to consider pancreatic dornase as the mucolytic agent of choice.
\end{abstract}

\section{Speculation}

Topical medications have been used on mucous membrane surfaces with little regard for whether the ciliary system is affected by them. Since all but one of the agents tested here showed pronounced ciliotoxic effect, we suggest that all topical drugs should undergo testing in a physiologic system such as chicken tracheal organ cultures.

A large number of topical medications are frequently used on mucous membrane surfaces. Although their extensive use as prescription and proprictary drugs would imply human safety, there is little available physiologic data related to their effect on cilia function. Because of this we have utilized the chicken tracheal organ culture system of Cherry and Taylor-Robinson (3) in the study of two topical decongestants and four mucolytic agents.

\section{MATERIALS AND METHODS}

In brief, tracheas were removed from 19-20-day-old chicken embryos (Red Ring Hatchery, Los Angeles), cut into ring sections $0.5-1.0 \mathrm{~mm}$ thick, and placed within $16 \times 125 \mathrm{~mm}$ screwcapped culture tubes (Corning. Corning, N.Y.), with the ring adhering to the surface of the tube $3-5 \mathrm{~cm}$ from the bottom. The tubes contained $1 \mathrm{ml}$ Eagle's basal medium (BME, Grand Island Biological, Grand Island, N. Y.) buffered by $N$-2-hydroxyethylpiperazine- $N^{\prime}$-2-ethanesulfonic acid (HEPES, Sigma Chemical Co., St. Louis, Mo.). The media had a pl of 7.0 and contained no serum. The cultures were incubated at $37^{\circ}$ on a roller drum (New Brunswick Scientific, New Brunswick. N. J.), one revolution every $5 \mathrm{~min}$, and ciliary activity was observed microscopically and graded as to its extent and vigor. The results were recorded as percentage of ciliary activity (2-4).

Tracheal rings were exposed to the experimental drugs by two techniques. In the first method $0.4 \mathrm{ml}$ test drug or control BME was added to each of five culture tubes and incubated for $5 \mathrm{~min}$ on the roller drum. After this, the drug containing medium was removed, the culture was washed once with BME, $1 \mathrm{ml}$ fresh BNE was added, and the tube was returned to the roller drum. This procedure was done three times daily for 5 daly's.

In the second laboratory method, ring sections were placed in the wells of microtiter plates (Limbro, Microbiological Associates. Bethesda, Md.), and covered with either test drug or BME for control. Five tracheal rings were used for each test substance. Ciliary activity was observed with an inverted microscope.

The following medications were tested: proprictary phenylephrine hydrochloride nasal spray $0.25 \%$ (Neosynephrine, Winthrop Lab.. New York, N. Y.) oxymetazoline hydrochloride $0.05 \%$ (Afrin, Schering, Kenilworth, N. J.), sodium bicarbonate $7.5 \%$ (Ivenex, Chagrin Falls. Ohio), acetyleysteine 10\% (Mead Johnson, Evansville, Ind.), pancreatic dornase 50.000) units/ml (Merck, Sharp and Dohme, West Point, Pa.), and Larginine $5.6 \%$ (L-arginine free base $3.66 \mathrm{~g}$; L-arginine hydrochloride $60.0 \mathrm{~g}$; sterile distilled water q.s. ad $1.200 \mathrm{ml}$ prepared by the pharmacy at UCLA Hospital).

\section{RESULTS}

The two topical decongestants caused a very rapid essation of ciliary activity when exposed to the tracheal rings in the wells of a microtiter plate (Table 1). Five minutes after immersion of the rings in medication, there was no remaining ciliary activity.

In Table 2 the results of similar experiments with mucolytic agents are presented. Sodium bicarbonate and acetyleysteine both produced complete loss of ciliary activity within 2 min. In contrast. at the end of $1 \mathrm{hr}$ there was $22 \%$ ciliary activity remaining in the L-arginine-exposed cultures and $96 \%$ activity left in the pancreatic dornase-immersed tracheal rings.

The effect on ciliary activity of the repeated application (three 5 -min exposures/day for 5 days) of topical decongestants to chicken tracheal organ cultures maintained in tubes is presented in Table 3. Complete cessation of ciliary activity occurred after two doses of phenylephrine and three doses of oxymetazoline. The results of similar experiments with mucolytic agents are presented in Figure 1. Two of the mucolytic agents (sodium

Table 1. Effect on ciliary activity of topical decongestants in chicke'n tracheal organ cultures observed in microtiter wells

\begin{tabular}{lcc}
\multicolumn{1}{c}{ Test drug } & \multicolumn{2}{c}{ Ciliary activity } \\
\cline { 2 - 3 } & $\begin{array}{c}\text { Before adminis- } \\
\text { tration }\end{array}$ & $\begin{array}{c}5 \text { min after ad- } \\
\text { ministration }\end{array}$ \\
\hline Oxymetazoline, $0.05 \%$ & 90 & 0 \\
Phenylephrine, $0.25 \%$ & 88 & 0 \\
Control BME & 98 & 98 \\
\hline
\end{tabular}


Table 2. Effect on ciliary activity of mucolytic agents in chicken tracheal organ cultures observed in microtiter wells

$\%$ Ciliary activity, time after administration

\begin{tabular}{|c|c|c|c|c|c|}
\hline \multirow[b]{2}{*}{ Test drug } & \multirow{2}{*}{$\begin{array}{c}\text { Before } \\
\text { administration }\end{array}$} & \\
\hline & & $2 \mathrm{~min}$ & $20 \mathrm{~min}$ & $30 \mathrm{~min}$ & $60 \mathrm{~min}$ \\
\hline Sodium bicarbonate, $7.5 \%$ & 96 & 0 & () & 0 & 0 \\
\hline Acetylcysteine, $10 \%$ & 90 & 0 & 0 & 0 & 0 \\
\hline L-Arginine, $5.6 \%$ & 80 & 85 & 86 & 60 & 22 \\
\hline Pancreatic dornase, 50,000 units $/ \mathrm{ml}$ & 100 & 100 & 95 & 95 & 96 \\
\hline Control BME & 100 & 100 & 97 & 96 & 95 \\
\hline
\end{tabular}

Table 3. Effect on ciliary activity of repeated application' of topical decongestants to chicken tracheal organ cultures maintained in tubes

\% Ciliary activity

\begin{tabular}{|c|c|c|c|c|c|}
\hline Test drug & $\begin{array}{l}\text { Before administra- } \\
\text { tion }\end{array}$ & After 1 st dose & After 2nd dose & After 3 rd dose & After 4th dose \\
\hline Oxymetazoline, $0.5 \%$ & 100 & 4 & 4 & 0 & () \\
\hline Phenylephrine, $0.25 \%$ & 98 & 19 & 0 & 0 & 0 \\
\hline Control BME & 100 & 96 & 90 & 90 & 9() \\
\hline
\end{tabular}

1 Three 5 -min exposures/day for 5 days.

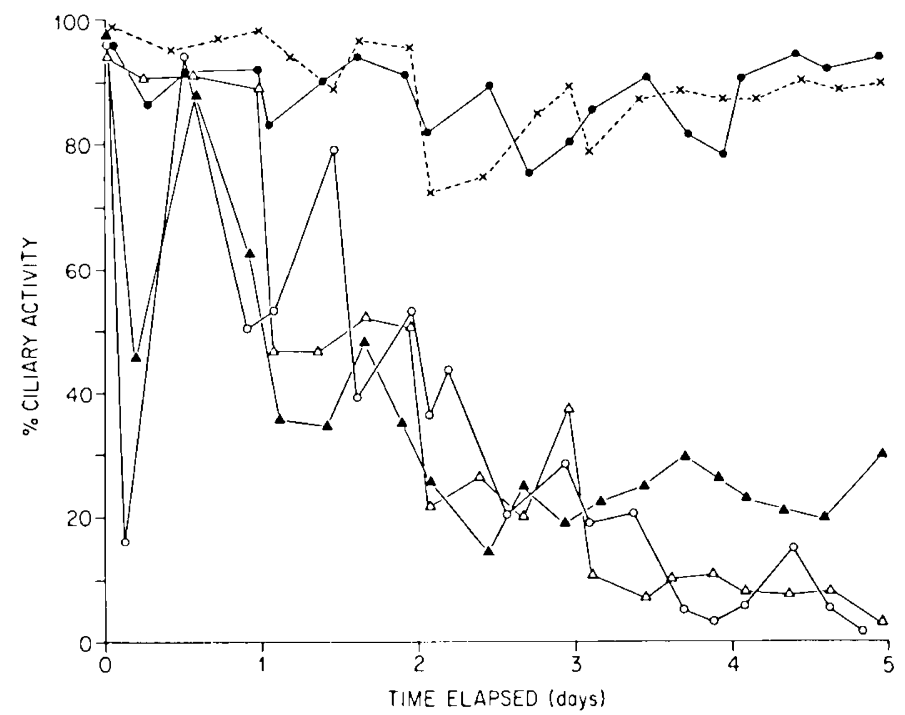

Fig. 1. The effect on ciliary activity of the repeated application (three 5-min exposures/day for 5 days) of mucolytic agents to chicken trachea organ cultures maintained in tubes: L-arginine, $5.6 \%(\triangle-\triangle)$; sodium bicarbonate, $7.5 \%(\bigcirc-\bigcirc)$; pancreatic dornase, 50,000 units $/ \mathrm{ml}$ $(\bullet-\longrightarrow)$; acetylcysteine, $5 \%(\mathbf{\Delta}-\mathbf{\Delta})$; control BME $(\times---\times)$.

bicarbonate and L-arginine) caused virtually a complete loss of ciliary activity over the 5-day study period. A $68 \%$ loss of ciliary activity resulted from the 15 acetylcysteine treatments. In contrast with the ciliary toxicity of sodium bicarbonate, L-arginine, and acetylcysteine, pancreatic dornase did not adversely affect ciliary activity.

\section{DISCUSSION}

The concentrations of the drugs utilized in this study are the ones normally used on mucous membrane surfaces. This effort, which was directed to see how well ciliated surfaces were able to withstand close contact with test drugs, took two forms: one, total immersion in the drug; the other, a relatively brief contact with the drug three times daily for 5 days. The cilia were unable to tolerate even brief immersion in four of the six test drugs. When contact with L-arginine was prolonged, ciliary activity was slowed substantially. Only pancreatic dornase demonstrated no toxicity during this kind of contact. This was no less true when multiple brief exposures were extended over 5 days. Using this organ culture system pancreatic dornase was the only test drug which had no adverse effect on the cilia in either form of exposure.

The organ culture system has been suggested as a useful method to evaluate ciliary function (13), for cilia are remarkably similar in structural detail wherever they are observed in the phylogenetic series (8). Not only are the results of ciliary activity in organ culture and in vivo comparable (5), but the cilia can be examined separately from the overlying mucous layer which some feel is propelled $(1,6)$ by a unidirectional metachronal planar movement of the cilia (5).

It is important to point out, however, that the pharmacologic agents evaluated in this study probably have their effect on the ciliated epithelial cells and not on the cilia per se. Considerable metabolic differences could exist between human and chicken cells. Therefore, some caution should be exercised in the direct extrapolation of chicken tracheal organ culture data to human toxicity. Also to be considered is the fact that the mucous layer is obviously disrupted in our organ culture system. It is therefore possible that the mucous layer offers some protection against the toxic effects of drugs on ciliated epithelium in vivo which is not present in the organ culture system (13).

The usefulness of topical decongestants on mucous membrane surfaces has never been proven. Because of the subjective improvement they provide, decongestants are frequently used despite the fact that the ciliotoxic properties of some decongestants have been demonstrated $(7,11,12)$. The uniformly bad effects seen on cilia by the two decongestants tested here suggest that they may be a poor choice for use on ciliated surfaces. In vitro the mucolytic properties of pancreatic dornase and acetylcysteine have been demonstrated (10). Although acetyleysteine liquifies both purulent and mucoid sputum in vitro, pancreatic cornase functions as a mucolytic agent only when DNA is present, that is, only in purulent sputum. (DNA is not present in mucoid sputum.)

Changing the viscosity of the mucous layer may not make clearance easier, for it has not been shown experimentally that a less viscous mucous layer is cleared more easily than a more tenacious layer. In fact there is some experimental evidence that indicates that addition of acetylcysteine slows the normal mucociliary rate $32 \%(9)$. In addition, there is always the possibility that if sputum is liquified in vivo airway resistance might be increased because of the "swelling" of the mucous layer (14). Thus, there is mixed experimental evidence regarding the usefulness of mucolytic agents. Now, in addition, there is the experi- 
mental evidence presented here demonstrating the ciliotoxic effect in the chicken tracheal organ culture system of three out of the four mucolytic agents tested.

\section{CONCLUSION}

Two nasal decongestants and four mucolytic agents were tested on the cilia of chicken tracheal organ cultures. Of all drugs tested only pancreatic dornase showed no ciliotoxicity. In the light of these results the use of the two nasal decongestants should be discouraged until further toxicity studies can be carried out in organ culture systems or in vivo. If the use of a mucolytic agents is felt to be necessary. pancreatic dornase should be the drug of choice.

\section{REFERENCES ANI NOTES}

1. Carson, S., Goldhamer, R., and Carpenter. R.: Mucous transport in the respiratory tract. Amer. Rev. Resp. Dis., 93: \$6 (1966)

2. Cherry, J. D., Roden, V. J., and Rejent, A. J.: The inhibition of ciliary activity in tracheal organ cultures by sera from children with cystic fibrosis and control subjects. J. Pediat., 79: 937 (1971).

3. Cherry, J. D., and Taylor-Robinson, D.: Large quantity production of chicken embryo tracheal organ cultures and use in virus and mycoplasma studies. Appl. Microbiol., 19: 658 (1970).

4. Cherry, J. D., and Taylor-Robinson, D.: Growth and pathogenesis of Mycoplasma mycoides var. capri in chicken embryo tracheal organ cultures. Infect. Immunity, 2: 431 (197(i).
5. Cheung. A. T. W., and Jahn. T. L.: Determination of the beat pattern of tracheal cilia. Pediat. Res., $10: 140$ (1976)

6. Dalhamn, T.: Mucous flow and ciliary activity in the trachea of healthy rats and rats exposed to respiratory irritant gases. Acta Physiol. Scand. Suppl., $123(1956)$.

7. Kimura, E. I': Effects of chemical agents on ciliated tracheal epithelium. Arch. Otolaryngol.. 69: 674 (1959).

8. Krahl, V. E., and Bulmash, M. H.: Studies on living ciliated epithelium. Amer. Rev. Resp. Dis., 99: 711 (1969).

9. Laurenzi, G. A.. Collins, B.. and Grearner, J. J.: Mucous flow in the mammalian trachea. Procecedings of the Tenth Aspen Emphysema Conference, pp. 27-40) (United States Public Health Service Publication no. 1787. 1967).

10. Licherman, J.: Measurement of sputum viscosity in cone-plate viscometer. Amer. Rev. Resp. Dis., 97: 662 (1968).

11. Lierle, D. M., and Mloore, P. M.: Effects of drugs on ciliary activity of mucosa of upper respiratory tract. Arch. Otolaryngol., $19: 55$ (1934).

12. Proetz. A. W.: Effects of certain drugs upon living ciliated epithelium. J. Laryngol. Otolaryngol., 49: 557 (19.34).

13. Rylander. R.: Current techniques to measure alterations in the ciliary activity of intact respiratory epithelium. Amer. Rev. Resp. Dis., 93: 67 (1966).

14. Taussig, L.: Mlists and aerosols: New studies and new thoughts. J. Pediat, 84 $619(1974)$.

15. We wish to acknowledge the assistance of Stephanie Biggs.

16. This research was presented in part at the Cystic Fibrosis Club Meeting, April 27, 1976 .

17. Requests for reprints should be addressed to: J. D. Cherry, M.D., Department of Pediatrics. University of California at Los Angeles, Los Angeles. Calif. 90024 (USA).

18. Received for publication October 21.1976.

19. Accepted for publication January 19, 1977
Enterocolitis milk leukocytes mononuclear phagocytes neonate phagocytosis

\title{
Protection against Experimental Necrotizing Enterocolitis by Maternal Milk. I. Role of Milk Leukocytes
}

\author{
JANE PITT, (3) BARBARA BARLOW, AND WILLIAN C. HEIRD \\ Departments of Pediatrics and Surgery, College of Physicians and Surgeons, Columbia University, \\ New York, New York, USA
}

\begin{abstract}
Summary
A rat model of necrotizing enterocolitis of the neonate in which maternal milk had been protective was studied to determine what components of the milk afforded protection and by what mechanism. Frozen and thawed rat milk was not protective, but formula supplemented with rat milk cells was. It was concluded that the cells provided protection. The cells, which are principally mononuclear phagocytes, can phagocytize and kill the Klebsiella pneumoniae strain used in the animal model. Animals with necrotizing enterolitis had peritonitis and bacteremia caused by this bacillus.
\end{abstract}

\section{Speculation}

Milk mononuclear phagocytes may protect the neonatal rat from enterocolitis by their antibacterial and wound-healing activities or by their ability to enhance the neonatal immune response. The etiologic role of bacteria in this disease, not proven in these experiments, must be determined by experiments in germfree rats.

Breast milk affords the neonatal animal protection against microbial infections (6-8, 14). Specific immunity has been attributed to antibodies (13). Additionally, antimicrobial activity of milk has been shown to reside in lactoferrin, Escherichia coli, lysozyme, and lactoperoxidase. Lactoferrin is bacteriostatic against E. coli (5) and Candida albicans (10), lysozyme is bactericidal against certain gram-positive organisms and may enhance complement-mediated bacterialysis of gram-negative bacteria (9), and lactoperoxidase is found in milk with hydrogen peroxide and halide and can kill bacteria (11). In addition, the high lactose, low protein, and low phosphate content of milk provides a poorly buffered medium which may discourage outgrowth of certain microorganisms. 